\title{
Validation of use of wireless monitors to measure levels of mobility during hospitalization
}

\author{
Cynthia J. Brown, MD, MSPH; ${ }^{1-2 *}$ David L. Roth, PhD; ${ }^{1,3}$ Richard M. Allman, MD $^{1-2}$ \\ ${ }^{1}$ Birmingham/Atlanta Department of Veterans Affairs Geriatric Research, Education, and Clinical Center, Birming- \\ ham, $A L$; Departments of ${ }^{2}$ Medicine and ${ }^{3}$ Biostatistics, University of Alabama at Birmingham, Birmingham, $A L$
}

\begin{abstract}
Accurate methods of measuring levels of mobility during hospitalization are lacking. We validated the use of wireless monitors to measure three levels of mobility during hospitalization: (1) lying, (2) sitting, and (3) standing or walking. Hospitalized medical patients 65 years or older who did not have delirium or dementia and could walk in the 2 weeks before admission were eligible. Wireless monitors, which recorded average position every 20 seconds, were attached to the thigh and ankle of consented patients. Direct behavioral observation by trained observers who recorded mobility level was used as the gold standard. Forty-seven male patients (mean age 73.9 years), with a mean length of stay of 5.3 days, generated 187 two-hour direct behavioral observation periods. Wireless monitors were highly correlated with direct behavioral observations. The median kappa was 0.92 and the kappa across all observations was 0.88 , indicating excellent agreement. This study demonstrates that wireless monitors validly measure mobility levels among older hospitalized patients.
\end{abstract}

Key words: aged, behavioral observation, frail elderly, geriatrics, hospitalization, recovery of function, rehabilitation, reproducibility of results, validation studies, walking.

\section{INTRODUCTION}

For older patients, hospitalization has been associated with a variety of adverse outcomes, including high rates of functional decline, increased risk of medical and iatrogenic events, and increased likelihood of nursing home placement upon discharge [1-10]. Low mobility, defined as activity that is limited to a bed or chair, is also a common event during hospitalization. An estimated 23 to 33 percent of older patients are limited to a bed or chair during hospitalization for acute illness [1,10-11]. Ambulation during hospitalization occurs infrequently, with only 27 percent of patients walking in the hallways during hospitalization [12]. Studies suggest that low mobility during hospitalization may be more related to aspects of hospital care such as immobilizing devices like catheters and intravenous lines than to the patients' admission diagnosis [7,13]. Importantly, low mobility has been associated with functional decline and new nursing home placement even after controlling for illness severity and comorbid illness [1].

Previous studies examining the prevalence of low mobility have been based on chart review, brief surveys of patient location, or every-other-day nursing reports $[1,10$ 11]. Researchers have assessed ambulation by observing

Abbreviations: $\mathrm{ADL}=$ activity of daily living, APACHE II = Acute Physiology and Chronic Health Evaluation II, BVAMC = Birmingham Department of Veterans Affairs Medical Center, $\mathrm{CAM}=$ Confusion Assessment Method, GPS = global positioning system, MMSE = Mini-Mental State Examination, PDA = personal digital assistant, $\mathrm{PI}=$ principal investigator, $\mathrm{SD}=$ standard deviation, VA = Department of Veterans Affairs.

* Address all correspondence to Cynthia J. Brown, MD, MSPH; University of Alabama at Birmingham, VAMC GRECC 11-G Room 8226, 1530 3rd Avenue South, Birmingham, AL 35294-0001; 205-934-9261; fax: 205-558-4749.

Email: cbrown@aging.uab.edu

DOI: 10.1682/JRRD.2007.06.0086 
the hallways for patients who are walking during a specific period of time [12]. Several limitations are inherent with these methods of measuring mobility. Mobility, particularly transferring or walking, is often a brief activity easily missed by nursing staff or surveys of location. This limitation is particularly true if the patient is independent with the activity. Chart documentation of mobility is often missing. And as previous research shows, few patients leave their rooms to walk [12], although they may be ambulatory within their rooms. The inability to measure the level of mobility throughout the entire hospital course has been a major barrier to mobility research in the hospital setting.

To date, no study has validated a method of measuring mobility that captures the level of mobility that occurs throughout hospitalization based on 24-hour data. As technology has improved, a variety of potential methods has become available, including global positioning systems (GPSs) [14-15], video recording [16], and small wireless accelerometers [17-18]. Unfortunately, the patient may consider video too intrusive, especially during hospitalization, and a GPS records where a person is in the environment not the level of mobility that is occurring. However, wireless accelerometers have been used previously to measure turning in bed [17]. These monitors can measure a patient's position based on movement and position of the limbs both in and out of bed and could potentially measure the different levels of mobility that occur during hospitalization.

This study validated the capability of wireless monitors to measure three levels of mobility during hospitalization: (1) lying, (2) sitting, and (3) standing or walking. We used direct behavioral observations as the gold standard for defining these three levels of mobility in order to (1) validate the use of the wireless monitors to measure the time that hospitalized patients spent at each mobility level and (2) compare the concordance of the wireless monitor with the direct observations when defining the specific level of mobility during a 20-second observation period.

\section{METHODS}

\section{Participants}

The participants were 47 patients aged $\geq 65$ years who were admitted to the medical wards of the Birmingham Department of Veterans Affairs (VA) Medical Center (BVAMC) from February 2006 to February 2007. A trained research assistant performed a brief screening interview with the physician to establish the presence of key screening inclusion criteria, including age $\geq 65$ years, a medical (vs surgical) reason for admission, and no isolation precautions. If a patient was eligible, consent to interview the patient was requested from the physician before the patient was contacted. Patients were approached regarding their interest in participating in the study. If they were interested, we evaluated the presence of delirium and dementia with the Confusion Assessment Method (CAM) [19] and the Mini-Mental State Examination (MMSE) [20], respectively. Self-reported ambulation status 2 weeks before admission was assessed to determine whether inclusion criteria were met. Inclusion criteria included-

1. MMSE score $\geq 16$.

2. No delirium (CAM score $=0$ ).

3. Self-report of being ambulatory with or without an assistive device in the 2 weeks before admission.

4. No significant language barrier requiring a translator.

5. No isolation precautions.

6. No previous enrollment in the study.

The first three criteria were chosen to select a group of veterans who would be more likely to be ambulatory during hospitalization, because our goal was to test the monitor's ability to accurately measure all three levels of mobility (i.e., lying, sitting, and standing or walking). Because of concerns about adequate sterilization of the monitors, we elected to exclude patients on isolation precautions.

Informed consent and all study protocols were approved by the institutional review board of the BVAMC. From February 2006 to February 2007, 69 eligible patients were admitted, with 19 declining to participate. Of the 50 who consented, 3 were not used in this analysis because of a lack of data from both the wireless monitor and direct behavioral observation. After initiating the observations, the principal investigator (PI) withdrew two patients because of declines in health status. Two patients withdrew after enrollment and an observation period, with both reporting that they disliked having someone observing them in the hospital room.

\section{Assessments}

In addition to the MMSE [20] and CAM [19], the baseline patient interview included a self-report of physical function in the 2 weeks before admission and the University of Alabama at Birmingham Study of Aging LifeSpace Assessment [21-22]. The Life-Space Assessment is a measure of mobility within the home and community and reflects physical performance and sociodemographic factors like age and transportation difficulties. Life-space can be conceptualized as a series of concentric areas that 
radiate from the place where the person sleeps. These areas range from being confined to one's bedroom to traveling outside one's community. The scores range from 0 to 120 , with higher scores indicating more mobility within the community. A brief functional assessment of the patient's ability to turn in bed, sit on the side of the bed, stand bearing weight, and walk a short distance was also evaluated [23]. The patient's ability to perform these tasks was graded with a graduated protocol that is standard for the Minimum Data Set assessment of function. The level of assistance is graded from a request for action (e.g., "please sit up on the side of the bed") to unable to perform the action without physical assistance [23]. This measure was chosen because it is standard in the nursing home setting and was relatively easy for even weak or sick patients to perform. A baseline nurse interview included a rating of the patient's ability to perform six basic activities of daily living (ADLs) upon hospital admission: feeding, bathing, dressing, toileting, transferring, and walking [24]. For each ADL, nurses were asked whether the patient was independent, required some assistance, or required total assistance. Medical records were extracted for admission data needed to calculate the High-Risk Diagnoses for the Elderly Scale [25] and the Acute Physiology and Chronic Health Evaluation II (APACHE II) score [26]. The HighRisk Diagnoses for the Elderly Scale uses a group of 10 high-risk medical diagnoses and weights them based on the magnitude of 1-year mortality risk. The scale potentially ranges from 0 to 27 , with $0=$ low risk, $1-2=$ intermediate risk, $3-5=$ high risk, and $>6=$ very high risk for 1-year mortality. The mortality rates associated with these four risk categories were $9.5,31,46$, and 73 percent, respectively. The High-Risk Diagnoses for the Elderly Scale has been validated and was a stronger predictor of mortality than several other measures, including the widely used Deyo-adapted Charlson index [25]. APACHE II assesses the acute severity of illness based on 12 physiological variables. Scores can range from 0 to 71, with a higher score indicating a more severe illness [26].

\section{Wireless Monitoring}

We used a large Band-Aid to attach wireless monitors (AugmenTech, Inc; Pittsburgh, Pennsylvania) to the patient's ipsilateral thigh and ankle. The wireless monitor is approximately 1 -inch square by $1 / 4$-inch deep and has been validated as a measure of bed mobility in nursing home residents [17]. The device measures horizontal and vertical orientation with respect to gravity every second and averages these data every 20 seconds, with the recording interval being programmable. The data are stored in the device and collected via a low-power radio link to a handheld computer. Previous pilot testing has indicated that the device is accurate within 2.5 to 5.0 degrees, which was deemed adequate for our study. The monitors were worn for 7 days or until the patient was discharged, whichever came first. Patients were visited daily to have the monitor moved to the contralateral leg and to have their skin examined for erythema or other signs of skin breakdown under the area of the monitor. Data were downloaded daily from the devices.

The data created by the monitors included vertical and horizontal position with respect to gravity and range over which the monitor had moved during the previous 20 -second epoch for the horizontal and vertical planes. For this study, we used only the vertical plane measurements from the thigh and ankle to determine a patient's position. We determined a priori an algorithm for identifying the three mobility levels of interest, lying, sitting, and standing or walking based on pilot data from University Hospital, Birmingham, Alabama. ${ }^{*}$ For example, if the thigh and ankle both read 60, the patient was noted to be lying in bed based on the monitor data. If the thigh and ankle were both greater than 100, the patient was noted to be standing or walking.

\section{Direct Behavioral Observation}

To validate the capability of the wireless monitor device to measure the three levels of mobility, we used direct behavioral observation as the gold standard. A researcher observed a participant for defined behaviors, in this case lying, sitting, and standing or walking. Before initiation of the study, we used objective observable criteria to define the behaviors. For example, patients who sat reclined in a recliner were considered to be lying. Those who sat up on the side of the bed or in a chair were considered to be sitting. We tested the reliability of the observational data by having the raters simultaneously code behaviors on a video, which we also used as a training tool. The video duplicated the usual positions and activities seen during hospitalization and was based on previous pilot data and the PI's clinical experience. Interrater reliability was excellent, with coders being accurate within 8 seconds of each other when capturing a change in mobility; for example, changing positions from lying to sitting. No differences

\footnotetext{
*Cynthia J. Brown, MD, MSPH. Unpublished data, 2004.
} 
were noted in coding of the behaviors of interest (lying, sitting, and standing or walking). Any questions regarding how to code a behavior were documented in the patient's data chart along with the time of the behavior. These data were entered into the protocol used by the study. Retraining with the video occurred periodically throughout the year of data collection.

Over 2 consecutive days, patients were observed for up to six 2-hour observation periods during the first 3 days of enrollment. The observer sat unobtrusively in the patient's room. Conversation was only initiated by the patient. Behaviors, specifically lying, sitting, and standing or walking, were recorded on a preprogrammed personal digital assistant (PDA). The PDA used an event recorder that continuously captured a level of mobility, such as sitting, until one of the other two levels was keyed into the device. Other data on the PDA included the location of the patient (in the room or hallway or off the ward), whether he was assisted and by whom (e.g., nurse, physical therapist, or family), and whether he was using an assistive device (i.e., cane, walker, wheelchair). Table 1 presents an example comparison of the data produced by the monitor, the interpretation of that data based on the a priori-determined algorithm of levels of mobility, and the observed mobility level.

Data from the wireless monitors were collected continuously throughout the hospital stay. However, for the validation component presented, the direct behavioral observation data on lying, sitting, and standing or walking that occurred during each 2-hour observation period were compared with the data obtained by the wireless monitors for the same 2-hour time period.

\section{Statistical Analysis}

The characteristics of the study participants were described with appropriate descriptive statistics, including frequency, proportion, mean, standard deviation (SD), and median (Table 2). To examine correlations across subjects, we calculated the mean and SD for the proportion of time each individual patient spent at all three mobility levels (lying, sitting, and standing or walking) as assessed by direct behavioral observations and as measured with the wireless monitors. Pearson correlations between the proportion of time spent at each level of mobility based on behavioral observations and the data provided by the wireless monitors were obtained. Using $\kappa$ statistics, we compared the concordance of the assessments of a patient's specific level of mobility as defined by direct behavioral observations over 20-second time periods with the level of mobility as determined with the wireless monitor data. The $\kappa$ statistics examine the probability of agreement between the monitors and the observations and determine the extent to which that agreement is greater than expected by chance given the base rates of the categories being observed [27]. Two different scales for the strength of agreement for $\kappa$ have been proposed. However, for both scales, $\kappa>0.8$ is considered excellent or near perfect [28]. For this study, monitor and observation data were coded every 20 seconds and $\kappa$ agreement statistics were calculated across time for each individual participant. The median of these $\kappa$ values was then examined as an overall measure of concurrent validity. All analyses were performed with SAS statistical software, version 9.1 (SAS Institute, Inc; Cary, North Carolina).

Table 1.

Example comparison of hospitalized patient's mobility levels as measured by wireless monitor (on thigh and ankle) and by direct behavioral observation.

\begin{tabular}{cccc}
\hline Raw Thigh Data & Raw Ankle Data & $\begin{array}{c}\text { Monitor Interpretation } \\
\text { Based on A Priori Algorithm }\end{array}$ & Observation of Mobility \\
\hline 44 & 74 & Lying & Lying \\
54 & 103 & Sitting & Standing/Walking \\
107 & 121 & Standing/Walking & Standing/Walking \\
75 & 114 & Sitting & Sitting \\
61 & 114 & Sitting & Sitting \\
61 & 114 & Sitting & Sitting \\
61 & 116 & Sitting & Sitting \\
107 & 120 & Standing & Standing \\
64 & 100 & Sitting & Sitting \\
\hline
\end{tabular}

*In this example, monitor and observation agree for all but this one 20-second monitor period. 


\section{RESULTS}

Characteristics of the cohort are presented in Table 2. The group had a mean \pm SD age of $73.9 \pm 6.5$ years, all were male, and 26 percent were African American. The APACHE II and High-Risk Diagnoses for the Elderly Scale indicated that the group had, on average, a moderate degree of illness severity and comorbidity. The mean \pm SD length of stay was $5.3 \pm 5.2$ days, with a range from 2 to 32 days. During the study, the PI withdrew two patients because they developed delirium. These patients who developed delirium subsequently died after an extended hospital stay.

There were 187 direct behavioral observation periods of 2 hours each for the 47 patients. Observation times for patients ranged from 120 minutes for those who completed only one 2-hour observation period to 720 minutes for those who completed six 2-hour direct observation periods. The mean total direct observation period for all 47 patients was 360 minutes. Proportion of time spent in each of the three mobility levels (lying, sitting, and standing or walking) was determined for each patient irrespective of the total time observed, allowing all participants to contribute equally to the correlations. Table 3 presents the correlation between the proportions of time spent at each level of mobility by participants as assessed by the wireless monitors during the times that the patients' mobility levels were also being assessed by direct behavioral observations.

Table 2.

Characteristics of study population $(N=47)$.

\begin{tabular}{|c|c|}
\hline Characteristic & Mean \pm SD or $n(\%)$ \\
\hline Age (yr) & $73.9 \pm 6.5$ \\
\hline Male & $47(100)$ \\
\hline African American & $12(26)$ \\
\hline Married & $22(47)$ \\
\hline MMSE Score & $24.9 \pm 2.9$ \\
\hline Comorbidity Score ${ }^{*}$ & $3.5 \pm 2.5$ \\
\hline APACHE II ${ }^{\dagger}$ & $10.6 \pm 3.6$ \\
\hline Life-Space Assessment Score ${ }^{\ddagger}$ & $64.2 \pm 26.9$ \\
\hline Length of Hospital Stay (d) & $5.3 \pm 5.2$ \\
\hline Received Physical Therapy & $16(34)$ \\
\hline Physician Ordered Bed Rest & $4(9)$ \\
\hline \multicolumn{2}{|c|}{$\begin{array}{l}{ }^{*} \text { As determined by High-Risk Diagnoses for the Elderly Scale; } 0=\text { low risk, } 1-2= \\
\text { intermediate risk, } 3-5=\text { high risk, and }>6 \text { = very high risk for } 1 \text {-year mortality. } \\
{ }^{\dagger} \text { Ranges from } 0-71 \text {, with higher scores indicating more severe illness. } \\
{ }^{\ddagger} \text { Ranges from } 0-120 \text {, with higher scores indicating better community mobility. } \\
\text { APACHE II = Acute Physiology and Chronic Health Evaluation II, MMSE = } \\
\text { Mini-Mental State Examination, SD = standard deviation. }\end{array}$} \\
\hline
\end{tabular}

The mean \pm SD proportion of minutes documented by direct behavioral observation for lying, sitting, and standing or walking was $71.3 \pm 28.6,25.3 \pm 25.7$, and $3.4 \pm$ 6.0 percent, respectively. Corresponding results obtained by the wireless monitors were $73.7 \pm 26.0,22.6 \pm 23.0$, and $3.7 \pm 5.7$ percent, respectively. The results obtained by the wireless monitors were highly correlated with the direct behavioral observations for the proportion of time spent in lying, sitting, and standing or walking, $r=0.98,0.97$, and 0.91 , respectively $(p<0.001)$ (Table 3$)$.

Of the 47 patients, $\kappa$ was estimated for 38 , with 9 being eliminated because of lack of variability. Specifically, they were noted to be lying by both the monitors and the observations for all their 2-hour observation periods. The individual $\kappa$ statistics ranged from 0.28 to 0.98 with a median $\kappa$ of 0.92 , indicating excellent agreement when categorizing patients at one of the three specific mobility levels. The monitors were valid for a majority of participants, with 29 patients (76\%) in the cohort having an individual $\kappa$ above 0.80 , the cut point for excellent agreement. The general $\kappa$ over all 20 -second observations and all patients was 0.88 (95\% confidence interval 0.8780.886), further supporting the validity of the wireless monitors.

\section{DISCUSSION}

These data suggest that wireless monitors can validly assess three specific mobility levels (i.e., lying, sitting, and standing or walking) during hospitalization. Patients tolerated wearing the monitors for up to 7 days without

Table 3.

Proportion of minutes (mean \pm standard deviation) hospitalized participants spent lying, sitting, and standing or walking when mobility levels were assessed both by direct behavioral observation and by wireless monitors $(N=47)$.

\begin{tabular}{lccc}
\hline $\begin{array}{c}\text { Mobility } \\
\text { Level }\end{array}$ & $\begin{array}{c}\text { Wireless } \\
\text { Monitors }\end{array}$ & $\begin{array}{c}\text { Behavioral } \\
\text { Observation }\end{array}$ & $\begin{array}{c}\text { Pearson } \\
\text { Correlation } \\
\text { Coefficient (r) }\end{array}$ \\
\hline Lying & $73.7 \pm 26.0$ & $71.3 \pm 28.6$ & 0.98 \\
Sitting & $22.6 \pm 23.0$ & $25.3 \pm 25.7$ & 0.97 \\
Standing or & $3.7 \pm 5.7$ & $3.4 \pm 6.0$ & 0.91 \\
$\quad$ Walking & & \\
${ }^{*} p<0.001$ for all mobility levels. \\
${ }^{\dagger}$ Total direct behavioral observation time ranged from 120-720 minutes for \\
individual patients (mean = 360 minutes). Proportion of time spent in each \\
mobility level was determined for each patient irrespective of total time \\
observed, allowing all participants to contribute equally to correlations. \\
\hline \hline
\end{tabular}


complaints of discomfort or evidence of skin problems. The monitors showed that, on average, patients spent 75 percent of each 2-hour observation period lying in bed.

Adverse outcomes associated with bed rest have been well described in studies sponsored by the National Aeronautics Space Association to evaluate the effects of space flight. For the young adults tested, orthostatic intolerance, decreased plasma volume of 5 to 10 percent, and average loss of muscle mass between 1 and 5 percent per day began within 24 hours of assuming the supine position [29]. The impact of low mobility on older patients is unknown; however, the adverse consequences of bed rest and low mobility are presumably amplified given this population's lower physiological reserves [30-32]. For example, sarcopenia, or muscle atrophy, is a known consequence of normal aging [33]. Many older adults are also deconditioned as a result of comorbid diseases and a sedentary lifestyle [33]. While the older adult is only hospitalized for an average of 5.6 days [34], this short hospital stay and its associated low mobility may translate into a significant loss of muscle strength and contribute to the functional decline observed after hospitalization.

Strengths of the study include the use of direct behavioral observation as a gold standard and the selection of patients who might be more active during hospitalization, thus testing the ability of the monitors to measure three levels of mobility: lying, sitting, and standing or walking.

Several important limitations warrant comment. First, we were unable to differentiate standing and walking in this study. The monitors were set to record every $20 \mathrm{sec}-$ onds. When patients walked, they would typically walk for 5 seconds, stand still for a few seconds, and then walk again. Very few patients actually walked in the hallway, a finding which has been previously described [12]. The monitors could accurately determine whether low mobility, defined as lying in bed or sitting up, occurred. Previous studies have correlated adverse outcomes with this definition of low mobility, so a measure that can accurately identify low mobility is important. A second limitation was the use of a human observer as the gold standard. Different observers possibly had different definitions of the levels of mobility, and the timing of triggering the event keys to indicate a change in activity may have differed. However, we addressed these concerns by intensively training the observers on videotaped activities similar to what would be experienced on the hospital wards and by correlating the results of the event coding among all the observers. In addition, all observers were taught clear definitions and any unusual activity was written into the data collection book and discussed by the observers, with consensus being reached about how to code the unusual behavior. Regardless of the method chosen to record the patient's mobility (i.e., an observer vs a video recorder in the room), a human observer must always assess the behavior and is therefore the best method available.

\section{CONCLUSIONS}

The clinical importance of low mobility, defined as lying in bed or sitting in a chair, for older adults during hospitalization is unknown. However, evaluating the importance of low mobility and examining the success of interventions to reduce low mobility require a valid method of examining the time that patients spend lying in bed, sitting up, and standing or walking. Wireless monitors provide a valid measure of these three specific mobility levels and permit accurate assessment of the length of time a person spends lying in bed or sitting in a chair. Previous research related to low mobility in the hospital has had to rely on chart reviews or nurse interviews to obtain crude, global assessments of mobility level.

The wireless monitors are easy to wear, noninvasive, and capture mobility continuously. This is the first study to examine the validity of a measure that can potentially capture the level of mobility achieved by a patient throughout his or her hospital stay and can be used in future studies on the impact of low mobility among hospitalized patients.

\section{ACKNOWLEDGMENTS}

We are indebted to the patients and nurses at the BVAMC who participated in this study. We wish to thank Kirsty J. Cutcliffe, Justin Miller, and Stephanie Stone for assistance with data collection; Eric V. Bodner for development of the PDA event program; and AugmenTech, Inc, for use of the wireless monitors.

An abstract of this research was presented at the annual meeting of the American Geriatrics Society (May 2007; Seattle, Washington).

This material was based on work supported in part by a Hartford Foundation-funded Southeast Center of Excellence in Geriatric Medicine grant (2004-0214) and a VA Research Career Development Award (E4-3842VA to Dr. Brown).

The authors have declared that no competing interests exist. 


\section{REFERENCES}

1. Brown CJ, Friedkin RJ, Inouye SK. Prevalence and outcomes of low mobility in hospitalized older patients. J Am Geriatr Soc. 2004;52(8):1263-70. [PMID: 15271112]

2. Covinsky KE, Palmer RM, Fortinsky RH, Counsell SR, Stewart AL, Kresevic D, Burant CJ, Landefeld CS. Loss of independence in activities of daily living in older adults hospitalized with medical illness: Increased vulnerability with age. J Am Geriatr Soc. 2003;51(4):451-58.

[PMID: 12657063$]$

3. Sager MA, Rudberg MA. Functional decline associated with hospitalization for acute illness. Clin Geriatr Med. 1998;14(4):669-79. [PMID: 9799473]

4. Hirsch CH, Sommers L, Olsen A, Mullen L, Winograd CH. The natural history of functional morbidity in hospitalized older patients. J Am Geriatr Soc. 1990;38(12):1296-1303. [PMID: 2123911]

5. Narain P, Rubenstein LZ, Wieland GD, Rosbrook B, Strome LS, Pietruszka F, Morley JE. Predictors of immediate and 6month outcomes in hospitalized elderly patients. The importance of functional status. J Am Geriatr Soc. 1988;36(9): 775-83. [PMID: 3411059]

6. Gorbein MJ, Bishop J, Beers MH, Norman D, Osterweil D, Rubenstein LZ. Iatrogenic illness in hospitalized elderly people. J Am Geriatr Soc. 1992;40(10):1031-42. [PMID: 1401678]

7. Gillick MR, Serrell NA, Gillick LS. Adverse consequences of hospitalization in the elderly. Soc Sci Med. 1982;16(10): 1033-38. [PMID: 6955965]

8. Rosin AJ, Boyd RV. Complications of illness in geriatric patients in hospital. J Chronic Dis. 1966;19(3):307-13. [PMID: 5910972]

9. Lamont CT, Sampson S, Matthias R, Kane R. The outcome of hospitalization for acute illness in the elderly. J Am Geriatr Soc. 1983;31(5):282-88. [PMID: 6841857]

10. Warshaw GA, Moore JT, Friedmans W, Currie CT, Kennie DC, Kane WJ, Mears PA. Functional disability in the hospitalized elderly. JAMA. 1982;248(7):847-50. [PMID: 6212699]

11. Lazarus BA, Murphy JB, Coletta EM, McQuade WH, Culpepper L. The provision of physical activity to hospitalized elderly patients. Arch Intern Med. 1991;151(12): 2452-56. [PMID: 1747003]

12. Callen BL, Mahoney JE, Grieves CB, Wells TJ, Enloe M. Frequency of hallway ambulation by hospitalized older adults on medical units of an academic hospital. Geriatr Nurs. 2004;25(4):212-17. [PMID: 15311196]

13. Inouye SK, Wagner DR, Acampora D, Horwitz RI, Cooney LM Jr, Hurst LD, Tinetti ME. A predictive index for functional decline in hospitalized elderly medical patients. J Gen Intern Med. 1993;8(12):645-52. [PMID: 8120679]
14. Lin CC, Chiu MJ, Hsiao CC, Lee RG, Tsai YS. Wireless health care service system for elderly with dementia. IEEE Trans Inf Technol Biomed. 2006;10(4):696-704. [PMID: 17044403]

15. Rodriguez DA, Brown AL, Troped PJ. Portable global positioning units to complement accelerometry-based physical activity monitors. Med Sci Sports Exerc. 2005;37(11 Suppl): S572-81. [PMID: 16294120]

16. Weinger MB, Gonzales DC, Slagle J, Syeed M. Video capture of clinical care to enhance patient safety. Qual Saf Health Care. 2004;13(2):136-44. [PMID: 15069222]

17. Bates-Jensen BM, Cadogan M, Jorge J, Schnelle JF. Standard quality-assessment system to evaluate pressure ulcer care in the nursing home. J Am Geriatr Soc. 2003;51(9): 1195-1202. [PMID: 12919230]

18. Culhane KM, O’Connor M, Lyons D, Lyons GM. Accelerometers in rehabilitation medicine for older adults. Age Ageing. 2005;34(6):556-60. [PMID: 16267178]

19. Inouye SK, van Dyck CH, Alessi CA, Balkin S, Siegal AP, Horwitz RI. Clarifying confusion: The Confusion Assessment Method. A new method for detection of delirium. Ann Intern Med. 1990;113(12):941-48. [PMID: 2240918]

20. Folstein MF, Folstein SE, McHugh PR. "Mini-mental state.” A practical method for grading the cognitive state of patients for the clinician. J Psychiatr Rev. 1975;12(3):189-98. [PMID: 1202204]

21. Baker PS, Bodner EV, Allman RM. Measuring life-space mobility in community-dwelling older adults. J Am Geriatr Soc. 2003;51(11):1610-14. [PMID: 14687391]

22. Parker M, Baker PS, Allman RM. A life-space approach to functional assessment of mobility in the elderly. J Gerontol Soc Work. 2001;35(4):35-56.

23. Department of Health and Human Services. Minimum Data Set, Version 3.0 (MDS 3.0) for nursing home resident assessment and care screening [monograph on the Internet]. Washington (DC): Department of Health and Human Services; 2006 [cited Apr 2007]. Available from: http:// www.cms.hhs.gov/nursinghomequalityinits/downloads/ MDS30Draft.pdf

24. Katz S, Ford AB, Moskowitz RW, Jackson BA, Jaffe MW. Studies of illness in the aged. The index of ADL: A standard measure of biological and psychosocial function. JAMA. 1963;185:914-19. [PMID: 14044222]

25. Desai MM, Bogardus ST Jr, Williams CS, Vitagliano G, Inouye SK. Development and validation of a risk-adjustment index for older patients: The High-Risk Diagnoses for the Elderly Scale. J Am Geriatr Soc. 2002;50(3):474-81. [PMID: 11943043]

26. Knaus WA, Draper EA, Wagner DP, Zimmerman JE. APACHE II: A severity of disease classification system. Crit Care Med. 1985;13(10):818-29. [PMID: 3928249] 
27. Agresti A. Categorical data analysis. 2nd ed. New York (NY): Wiley-Interscience; 2002. p. 434-35.

28. Feinstein AR. Principles of medical statistics. Boca Raton (FL): Chapman \& Hall/CRC; 2002. p. 417.

29. Fortney SM, Schneider VS, Greenleaf JE. The physiology of bedrest. In: Fregly MJ, Blatteis CM, editors. Handbook of physiology. Section 4: Environmental physiology. Bethesda (MD): American Physiology Society; 1996. p. 889-939.

30. Creditor MC. Hazards of hospitalization of the elderly. Ann Intern Med. 1993;118(3):219-23. [PMID: 8417639]

31. Harper CM, Lyles YM. Physiology and complications of bedrest. J Am Geriatr Soc. 1988;36(11):1047-54.

[PMID: 3049751]

32. Hoenig HM, Rubenstein LZ. Hospital-associated deconditioning and dysfunction. J Am Geriatr Soc. 1991;39(2):220-22. [PMID: 1991956]
33. Roubenoff R. Sarcopenia and its implications for the elderly. Eur J Clin Nutr. 2000;54 Suppl 3:S40-47. [PMID: 11041074$]$

34. Centers for Disease Control and Prevention. National Hospital Discharge Survey Data Highlights [monograph on the Internet]. Hyattsville (MD): U.S. Department of Health and Human Services, Centers for Disease Control and Prevention, National Center for Health Statistics; 2007 [cited May 2008]. Available from:

http://www.cdc.gov/nchs/about/major/hdasd/nhdstab.htm

Submitted for publication June 15, 2007. Accepted in revised form December 10, 2007. 